Göteborg ITP 93-19

August 1993

\title{
A note on path integrals and time evolutions in BRST quantization.
}

\author{
Robert Marnelius \\ Institute of Theoretical Physics \\ Chalmers University of Technology \\ S-412 96 Göteborg, Sweden
}

\begin{abstract}
Recent formal solutions of BRST quantization on inner product spaces within the operator method are shown to lead to an unexpected interpretation of the conventional path integral formulation. The relation between the Hamiltonians in the two formulations is nontrivial. For the operator method the correspondence requires certain quantum rules which make the formal solutions exact, and for the path integral the correspondence yields a precise connection between boundary conditions and the choice of gauge fixing.
\end{abstract}


The original development of the quantization procedure for gauge theories was made within the path integral formulation [1, 2, 3, ㄴ, The operator method came later [5, 6] and has been helpful to acquire a more precise understanding of the BRST quantization. Recently we have derived general formal solutions for a large class of BRST models on inner product spaces within the operator formulation [7, 8, 9]. As we shall see these solutions lead to an unexpected interpretation of the original path integral expressions. In addition, this connection gives information of how the formal solutions in [7, 8] are to be quantized, rules which are in agreement with those argued for in [9]. For the path integral expressions we obtain a precise connection between the choices of gauge fixing and the boundary conditions to be imposed.

The conventional connection between operator quantization and path integrals is through the time slice formula $(\hbar=1)$

$$
\begin{aligned}
& \left\langle\phi^{\prime}, t^{\prime} \mid \phi, t\right\rangle=\left\langle\phi^{\prime}\left|e^{-i\left(t^{\prime}-t\right) H}\right| \phi\right\rangle=\int d^{n} q^{\prime} d^{n} q \phi^{\prime *}\left(q^{\prime}\right) \phi(q)\left\langle q^{\prime}, t^{\prime} \mid q, t\right\rangle \\
& =\left\langle q^{\prime}, t^{\prime} \mid q, t\right\rangle=\int \prod_{m=1}^{N-1} d^{n} q_{m} \prod_{k=0}^{N-1}\left\langle q_{k+1}, t_{k+1} \mid q_{k}, t_{k}\right\rangle= \\
& =\int \prod_{m=1}^{N-1} d^{n} q_{m} \prod_{k=0}^{N-1} \frac{d^{n} p_{k}}{(2 \pi)^{n}} \exp \left(i p_{k} \cdot \Delta q_{k}-i \Delta t H\left(p_{k}, \bar{q}_{k}\right)\right) \\
& \stackrel{\lim _{N \rightarrow} \infty}{\longrightarrow} \int_{P a t h} \prod_{t} \frac{d^{n} q d^{n} p}{(2 \pi)^{n}} \exp i \int_{t}^{t^{\prime}} d t(p \cdot \dot{q}-H(p, q))
\end{aligned}
$$

where $q_{0}=q, \quad q_{N}, q_{\infty}=q^{\prime}, \quad t_{0}=t, \quad t_{N}, t_{\infty}=t^{\prime}, \quad \Delta q_{k}=q_{k+1}-q_{k}$ and $\bar{q}_{k}=$ $\frac{1}{2}\left(q_{k+1}+q_{k}\right) . H(p, q)$ is the Weyl transform of the Hamiltonian operator $H(P, Q)$ defined by

$$
\begin{aligned}
& H(p, q)=\frac{1}{(2 \pi)^{n}} \int d^{n} u d^{n} v \tilde{H}(u, v) e^{-i(q \cdot u+p \cdot v)} \\
& H(P, Q)=\frac{1}{(2 \pi)^{n}} \int d^{n} u d^{n} v \tilde{H}(u, v) e^{-i(Q \cdot u+P \cdot v)}
\end{aligned}
$$

which implies

$$
\left\langle q^{\prime \prime}|H(P, Q)| q^{\prime}\right\rangle=\int \frac{d^{n} p}{(2 \pi)^{n}} e^{i\left(q^{\prime \prime}-q^{\prime}\right) \cdot p} H(p, \bar{q})
$$

where $\bar{q} \equiv \frac{1}{2}\left(q^{\prime \prime}+q^{\prime}\right)$, a relation which is used in (11). (The canonical conjugate operators $Q_{i}$ and $P_{i}, i=1, \ldots, n$, are hermitian and satisfy $\left[Q_{i}, P_{j}\right]_{-}=i \delta_{i j}$, and $|q\rangle$ are eigenstates to $Q_{i}$ with real eigenvalues $q_{i}$.) 
Eqn (1) represents a connection between operator quantization and path integrals for ordinary quantum mechanics in which $|\phi, t\rangle$ belongs to a Hilbert space. In a gauge theoretic framework one considers a larger state space which also contains indefinite metric states. For such states hermitian coordinate and momentum operators do not have real spectra [10. Instead they have imaginary ones if a Hilbert space topology is imposed (which is natural). In such a case the eigenstates satisfy [11, 12, 13]

$$
\begin{aligned}
& Q|i q\rangle=i q|i q\rangle, \quad\langle-i q| \equiv(|i q\rangle)^{\dagger}, \quad\left\langle i q \mid i q^{\prime}\right\rangle=\delta^{n}\left(q-q^{\prime}\right) \\
& \int d^{n} q|i q\rangle\left\langle i q\left|=\int d^{n} q\right|-i q\right\rangle\langle-i q|=\mathbf{1}
\end{aligned}
$$

which modifies the time slicing formula (19). Instead of (3) we get

$$
\left\langle i q^{\prime \prime}|H(P, Q)| i q^{\prime}\right\rangle=\int \frac{d^{n} p}{(2 \pi)^{n}} e^{i\left(q^{\prime \prime}-q^{\prime}\right) \cdot p} H(-i p, i \bar{q})
$$

where $H(-i p, i \bar{q})$ is a real function when written in terms of real arguments if $H(P, Q)$ is hermitian. The path integral formula (1) is then turned into

$$
\left\langle\phi^{\prime}, t^{\prime} \mid \phi, t\right\rangle=\left\langle\phi^{\prime}\left|e^{-i\left(t^{\prime}-t\right) H}\right| \phi\right\rangle=\int d^{n} q^{\prime} d^{n} q \phi^{\prime *}\left(-i q^{\prime}\right) \phi(i q)\left\langle i q^{\prime}, t^{\prime} \mid i q, t\right\rangle
$$

where (see also [11, 12])

$$
\begin{aligned}
& \left\langle i q^{\prime}, t^{\prime} \mid i q, t\right\rangle=\int \prod_{m=1}^{N-1} d^{n} q_{m} \prod_{k=0}^{N-1}\left\langle i q_{k+1}, t_{k+1} \mid i q_{k}, t_{k}\right\rangle= \\
& =\int \prod_{m=1}^{N-1} d^{n} q_{m} \prod_{k=0}^{N-1} \frac{d^{n} p_{k}}{(2 \pi)^{n}} \exp \left(i p_{k} \cdot \Delta q_{k}-i \Delta t H\left(-i p_{k}, i \overline{q_{k}}\right)\right) \\
& \stackrel{\lim _{N \rightarrow} \infty}{\longrightarrow} \int_{\text {Path }} \prod_{t} \frac{d^{n} q d^{n} p}{(2 \pi)^{n}} \exp i \int_{t}^{t^{\prime}} d t(p \cdot \dot{q}-H(-i p, i q))
\end{aligned}
$$

The obvious problem with this formula is that the Hamiltonian $H(-i p, i q)$ is not real in general although the Hamiltonian operator $H(P, Q)$ is hermitian. Notice that the formulas are symmetric in the sense that (6) may also be written as

$$
\left\langle\phi^{\prime}, t^{\prime} \mid \phi, t\right\rangle=\int d^{n} q^{\prime} d^{n} q \phi^{\prime *}\left(i q^{\prime}\right) \phi(-i q)\left\langle-i q^{\prime}, t^{\prime} \mid-i q, t\right\rangle
$$

where the propagator $\left\langle-i q^{\prime}, t^{\prime} \mid-i q, t\right\rangle$ is given by (7) with $H(-i p, i q)$ replaced by $H(i p,-i q)$ which is its complex conjugate when $H(P, Q)$ is hermitian. Hence, 
$\left\langle\phi^{\prime}, t^{\prime} \mid \phi, t\right\rangle$ will grow exponentially both when $t \rightarrow \infty$ and $t \rightarrow-\infty$ if $H(i p,-i q)$ contains imaginary terms. To avoid this property one has to require that $H(i p,-i q)$ is real [11].

Consider now hermitian fermionic canonical conjugate operators $\hat{\mathcal{P}}_{i}$ and $\hat{\eta}_{i}, i=$ $1, \ldots, n$, satisfying $\left[\hat{\mathcal{P}}_{i},\left.\hat{\eta}_{j}\right|_{+}=\delta_{i j}\right.$. They span a finite dimensional indefinite metric state space. If one introduces odd Grassmann numbers one may derive pseudoclassical path integrals for theories involving $\hat{\mathcal{P}}_{i}$ and $\hat{\eta}_{i}$ and the $\operatorname{Hamiltonian} H(\hat{\mathcal{P}}, \hat{\eta})$. In fact there are two options: One may either make use of eigenstates with eigenvalues which are real odd Grassmann numbers, $\hat{\eta}_{i}|\eta\rangle=\eta_{i}|\eta\rangle$, or which are imaginary odd Grassmann numbers, $\hat{\eta}_{i}|i \eta\rangle=i \eta_{i}|i \eta\rangle$ ( $\eta_{i}$ is real and odd). In the first case one finds the path integral (the conventions of [14] is used and appropriate orderings for $n$ odd is ignored)

$$
\begin{aligned}
& \left\langle\eta^{\prime}, t^{\prime} \mid \eta, t\right\rangle=\int \prod_{m=1}^{N-1} d^{n} \eta_{m} \prod_{k=0}^{N-1}\left\langle\eta_{k+1}, t_{k+1} \mid \eta_{k}, t_{k}\right\rangle= \\
& =\int \prod_{m=1}^{N-1} d^{n} \eta_{m} \prod_{k=0}^{N-1} d^{n} \mathcal{P}_{k} \exp \left(-\mathcal{P}_{k} \cdot \Delta \eta_{k}-i \Delta t H\left(\mathcal{P}_{k}, \bar{\eta}_{k}\right)\right) \\
& \stackrel{\lim _{\mathrm{m}^{\prime}}}{\longrightarrow} \int_{\text {Path }} \prod_{t} d^{n} \eta d^{n} \mathcal{P} \exp i \int_{t}^{t^{\prime}} d t(i \mathcal{P} \cdot \dot{\eta}-H(\mathcal{P}, \eta))
\end{aligned}
$$

where $\eta_{0}=\eta, \quad \eta_{N}, \eta_{\infty}=\eta^{\prime}, \quad t_{0}=t, \quad t_{N}, t_{\infty}=t^{\prime}, \quad \Delta \eta_{k}=\eta_{k+1}-\eta_{k}$ and $\overline{\eta_{k}}=\frac{1}{2}\left(\eta_{k+1}+\eta_{k}\right) . H(\mathcal{P}, \eta)$ is the fermionic Weyl transform of the Hamiltonian operator $H(\hat{\mathcal{P}}, \hat{\eta})$ defined by

$$
\begin{aligned}
& H(\mathcal{P}, \eta)=\int d^{n} \lambda d^{n} \xi \tilde{H}(\lambda, \xi) e^{-\mathcal{P} \cdot \lambda-\eta \cdot \xi} \\
& H(\hat{\mathcal{P}}, \hat{\eta})=\int d^{n} \lambda d^{n} \xi \tilde{H}(\lambda, \xi) e^{-\hat{\mathcal{P}} \cdot \lambda-\hat{\eta} \cdot \xi}
\end{aligned}
$$

which implies

$$
\left\langle\eta^{\prime \prime}|H(\hat{\mathcal{P}}, \hat{\eta})| \eta^{\prime}\right\rangle=\int d^{n} \mathcal{P} e^{-\mathcal{P} \cdot\left(\eta^{\prime \prime}-\eta^{\prime}\right)} H(\mathcal{P}, \bar{\eta})
$$

where $\bar{\eta} \equiv \frac{1}{2}\left(\eta^{\prime \prime}+\eta^{\prime}\right)$. This relation is used in (9). (The last line in (9) is somewhat misleading for odd $n$ since the finite slice expression contains one more $\mathcal{P}$-integral than $\eta$-integral which makes $\left\langle\eta^{\prime}, t^{\prime} \mid \eta, t\right\rangle$ odd for odd $n$.) 
Using the same conventions as above the imaginary odd Grassmann eigenstates satisfy the properties

$$
\begin{aligned}
& \hat{\eta}|i \eta\rangle=i \eta|i \eta\rangle, \quad\langle-i \eta| \equiv(|i \eta\rangle)^{\dagger}, \quad\left\langle i \eta \mid i \eta^{\prime}\right\rangle=i^{n} \delta^{n}\left(\eta-\eta^{\prime}\right) \\
& \int|i \eta\rangle(-i)^{n} d^{n} \eta\left\langle i \eta\left|=\int\right|-i \eta\right\rangle i^{n} d^{n} \eta\langle-i \eta|=\mathbf{1}
\end{aligned}
$$

Instead of (11) we get now

$$
\left\langle i \eta^{\prime \prime}|H(\hat{\mathcal{P}}, \hat{\eta})| i \eta^{\prime}\right\rangle=i^{n} \int d^{n} \mathcal{P} e^{-\mathcal{P} \cdot\left(\eta^{\prime \prime}-\eta^{\prime}\right)} H(i \mathcal{P},-i \bar{\eta})
$$

which leads to the path integral

$$
\begin{aligned}
& \left\langle i \eta^{\prime}, t^{\prime} \mid i \eta, t\right\rangle=\int \prod_{m=1}^{N-1}(-i)^{n} d^{n} \eta_{m} \prod_{k=0}^{N-1}\left\langle i \eta_{k+1}, t_{k+1} \mid i \eta_{k}, t_{k}\right\rangle= \\
& =\int \prod_{m=1}^{N-1}(-i)^{n} d^{n} \eta_{m} \prod_{k=0}^{N-1} i^{n} d^{n} \mathcal{P}_{k} \exp \left(-\mathcal{P}_{k} \cdot \Delta \eta_{k}-i \Delta t H\left(i \mathcal{P}_{k},-i \bar{\eta}_{k}\right)\right) \\
& \stackrel{\lim _{N \rightarrow \infty}}{\longrightarrow} \int_{\text {Path }} \prod_{t} d^{n} \eta d^{n} \mathcal{P} \exp i \int_{t}^{t^{\prime}} d t(i \mathcal{P} \cdot \dot{\eta}-H(i \mathcal{P},-i \eta))
\end{aligned}
$$

(Also this expression is misleading for odd $n$.)

In [4] the following Hamiltonian form of the path integral for finite dimensional bosonic gauge theories were given (we suppress factors of $2 \pi$ in the following) $(2 m<$ $n)$

$$
\begin{aligned}
& Z_{\rho}=\int d^{n} q d^{n} p d^{m} v d^{m} \pi d^{m} \eta d^{m} \mathcal{P} d^{m} \bar{\eta} d^{m} \overline{\mathcal{P}} \times \\
& \times \exp i \int d t\left(p \cdot \dot{q}+\pi \cdot \dot{v}+i \mathcal{P} \cdot \dot{\eta}+i \overline{\mathcal{P}} \cdot \dot{\bar{\eta}}-H_{\rho}\right)
\end{aligned}
$$

where $\eta^{a}, \bar{\eta}_{a}$ and $v^{a}$ are ghosts, antighosts and Lagrange multipliers respectively, and where

$$
H_{\rho} \equiv H_{0}+\{\rho, Q\}
$$

is a BRST invariant Hamiltonian. $Q$ is the BRST charge, $H_{0}$ is BRST invariant and $\rho$ is a real odd gauge fixing function. In (16) $\rho$ has typically the form $\mathcal{P}_{a} v^{a}$ or/and $\bar{\eta}_{a} \chi^{a}$. (It must have ghost number minus one.) Usually it turns $H_{\rho}$ into a form which allows for an integration over the momenta in $Z_{\rho}$ such that

$$
Z_{\rho}=\int D^{n} q D^{m} v D^{m} \eta D^{m} \bar{\eta} \exp i \int d t L(t)
$$


where $L(t)$ is a regular configuration space Lagrangian. (Contrary to (15) the configuration space expression (17) usually involves a nontrivial measure.)

In the corresponding operator formulation of the BRST quantization in (15) one starts with a large state space $\Omega$ spanned by the canonical operators $(P, Q),(\hat{\pi}, \hat{v})$, $(\hat{\mathcal{P}}, \hat{\eta})$ and $(\hat{\overline{\mathcal{P}}}, \hat{\bar{\eta}})$. One defines the BRST charge operator $\hat{Q}$ in such a way that it is nilpotent $\left(\hat{Q}^{2}=0\right)$, and one projects out the physical state space $\Omega_{p h}$ by $\hat{Q}|p h\rangle=0$ which is a regular state space if all zero norm states of the form $\hat{Q}|\chi\rangle$ are divided out. What is the Hamiltonian operator? From (16) it is natural to choose

$$
\hat{H}_{\rho}=\hat{H}_{0}+[\hat{\rho}, \hat{Q}]_{+}
$$

which is hermitian. This choice seems to comply with the Fradkin-Vilkovisky theorem [3, 田, 15] which says that the path integral expression (15) is independent of $\rho$, since the second term in (18) only seems to produce zero norm states on physical states and which therefore may be divided out. However, this is in general not true since

$$
[\hat{\rho}, \hat{Q}]_{+}|p h\rangle=\hat{Q} \hat{\rho}|p h\rangle
$$

is only a zero norm state if it also belongs to an inner product space. This implies that the above formal arguments have to be replaced by more precise ones.

The operator formulation of the BRST quantization on inner product spaces was considered in [7, [8] for gauge theories with finite number of degrees of freedom with a nilpotent BRST charge of the BFV form [4]

$$
\hat{Q}=\hat{\psi}_{a} \hat{\eta}^{a}-\frac{1}{2} i U_{b c}{ }^{a} \hat{\mathcal{P}}_{a} \hat{\eta}^{b} \hat{\eta}^{c}-\frac{1}{2} i U_{a b}{ }^{b} \hat{\eta}^{a}+\hat{\mathcal{P}}_{a} \hat{\pi}^{a}
$$

where $\hat{\psi}_{a}$ are hermitian bosonic gauge generators (constraints) satisfying the Lie algebra

$$
\left[\hat{\psi}_{a}, \hat{\psi}_{b}\right]_{-}=i U_{a b}^{c} \hat{\psi}_{c}
$$

where $U_{a b}^{c}$ are the structure constants. By means of a bigrading [16] general solutions of $\hat{Q}|p h\rangle=0$ were derived all of the form (apart from zero norm states)

$$
|p h\rangle=e^{\alpha[\hat{\rho}, \hat{Q}]+}|\phi\rangle
$$


where $|\phi\rangle$ is a BRST invariant state and where $\alpha$ is a real parameter different from zero. In [7]

$$
\hat{\rho}=\hat{\mathcal{P}}_{a} \hat{v}^{a}
$$

and $|\phi\rangle$ satisfies

$$
\hat{\pi}_{a}|\phi\rangle=\hat{\eta}^{a}|\phi\rangle=\hat{\bar{\eta}}_{a}|\phi\rangle=0
$$

which makes $|\phi\rangle$ BRST invariant. Notice that although $|\phi\rangle$ does not belong to an inner product space $|p h\rangle$ will do provided the quantization is appropriately prescribed. Thus, although we formally have

$$
|p h\rangle=|\phi\rangle+\hat{Q}|\cdot\rangle
$$

we may not divide out $\hat{Q}|\cdot\rangle$. In [8] another set of solutions were derived. They are of the form (22) but with

$$
\hat{\rho}=\hat{\bar{\eta}}_{a} \hat{\chi}^{a}
$$

where $\hat{\chi}^{a}$ is a hermitian gauge fixing operator to the gauge generators $\hat{\psi}_{a} \cdot\left(\left[\hat{\chi}^{a}, \hat{\psi}_{b}\right]_{-}\right.$ must be nonsingular.) Instead of (24) $|\phi\rangle$ satisfies here

$$
\hat{\mathcal{P}}_{a}|\phi\rangle=\hat{\overline{\mathcal{P}}} a|\phi\rangle=\left(\hat{\psi}_{a}+i U_{a b}{ }^{b}\right)|\phi\rangle=0
$$

which also makes $|\phi\rangle$ BRST invariant.

Consider now the physical transition amplitude

$$
\begin{aligned}
& \left\langle p h^{\prime}, t^{\prime} \mid p h, t\right\rangle=\left\langle p h^{\prime}\left|e^{-i\left(t^{\prime}-t\right) \hat{H}_{0}}\right| p h\right\rangle= \\
& =\left\langle\phi^{\prime}\left|e^{\alpha[\hat{\rho}, \hat{Q}]} e^{-i\left(t^{\prime}-t\right) \hat{H}_{0}} e^{\alpha[\hat{\rho}, \hat{Q}]}\right| \phi\right\rangle=\left\langle\phi^{\prime}\left|e^{-i\left(t^{\prime}-t\right) \hat{H}_{0}+2 \alpha[\hat{\rho}, \hat{Q}]}\right| \phi\right\rangle
\end{aligned}
$$

where $\hat{H}_{0}$ is a BRST invariant Hamiltonian operator. The last equality is valid provided $H_{0}$ commutes with $[\hat{\rho}, \hat{Q}]$ which we assume. ([$\left.\hat{\rho}, \hat{H}_{0}\right]$ must be BRST invariant.) In [7, 8] it was shown that (28) is independent of the value of the real parameter $\alpha$ when $t^{\prime}=t$ except that it must be nonzero. This should also be the case for $t^{\prime} \neq t$. We may therefore set $2 \alpha= \pm\left(t^{\prime}-t\right)$ except when $t^{\prime}=t$. Eqn (28) looks then like a 
transition amplitude for the physical states $|\phi\rangle$ where time translation is generated by the non-hermitian Hamiltonian operator

$$
\hat{H}_{\rho}^{c}=\hat{H}_{0} \pm i[\hat{\rho}, \hat{Q}]
$$

The conventional identification (18) leads to an imaginary $\alpha$ (see [20, 18, 19]). which is not allowed according to [7, 8]. Eqn (29) seems therefore to be in contradiction with the formulas (15) and (16). However, it is at this point indefinite metric state spaces and imaginary eigenvalues will help us to resolve this contradiction. In [9] (see also [13]) quantization rules are proposed which tell us which variables may be quantized with indefinite metric states and which may not. For bosonic gauge theories it was argued that the Lagrange multipliers should be quantized with opposite metric states to the variable which the gauge generators $\psi_{a}$ eliminate. These rules lead to certain consequences for the additional term to the Hamiltonian $H_{0}$. Notice that

$$
\begin{aligned}
& {[\hat{\rho}, \hat{Q}]=\left(\hat{\psi}_{a}+\hat{\psi}_{a}{ }^{g h}\right) \hat{v}^{a}-i \hat{\mathcal{P}}_{a} \hat{\overline{\mathcal{P}}}^{a}} \\
& \hat{\psi}_{a}^{g h}=\frac{1}{2} i U_{a b}{ }^{c}\left(\hat{\mathcal{P}}_{c} \hat{\eta}^{b}-\hat{\eta}^{b} \hat{\mathcal{P}}_{c}\right)
\end{aligned}
$$

for $\hat{\rho}=\hat{v}^{a} \hat{\mathcal{P}}_{a}$, and that

$$
[\hat{\rho}, \hat{Q}]=\hat{\pi}_{a} \hat{\chi}^{a}+\hat{\bar{\eta}}_{a} \hat{\eta}^{b}\left[\hat{\chi}^{a}, \hat{\psi}_{b}\right]
$$

for $\hat{\rho}=\hat{\bar{\eta}}_{a} \hat{\chi}^{a}$. We have then from (6) and (7) that $[\hat{\rho}, \hat{Q}]$ leads to an imaginary expression in the path integral if the Lagrange multipliers $(\hat{\pi}, \hat{v})$ are quantized with indefinite metric states and if imaginary eigenstates are chosen for the fermionic ghosts $(\hat{\mathcal{P}}, \hat{\eta})$ or the antighosts $(\hat{\overline{\mathcal{P}}}, \hat{\bar{\eta}})$ which always is possible. Under these conditions the transition amplitude (28) is consistent with the path integral (15) and (16) provided $\hat{H}_{0}$ leads to a real expression. Since the general solutions (22) are expected to be valid also for graded symmetries we may directly generalize the above quantization rules to such theories. They imply that bosonic ghosts and antighosts are to be quantized with opposite metric states in agreement with the proposal in [9] and that imaginary eigenstate representations are to be chosen for odd Lagrangian 
multipliers. A further implication is that theories with $H_{0}=0$ are invariant under time reversal since we may choose either sign in (29) and in the path integrals.

Another possibility is to consider the transition amplitude (28) for imaginary times $t=-i \tau$. In this case we may immediately identify the hermitian Hamiltonian operator (18) from the choice $2 \alpha=-\left(\tau^{\prime}-\tau\right)$. It may lead to a real Hamiltonian in the path integral provided the Lagrange multipliers are quantized with positive metric states and if the variable which $\psi_{a}$ eliminates is quantized with negative metric states and $\psi_{a}$ remains real. The latter case occurs if e.g. $\psi_{a}$ is quadratic in the canonical conjugate variable to the variable which it eliminates. This possibility is e.g. used when a Euclidean propagator is derived from the spinless particle model in [9]. However, particles with spin lead to complex Hamiltonians in the path integral. When these Euclidean propagators are analytically continued to Minkowski space the corresponding path integrals seem more to comply with the conventional picture (15) with real Hamiltonians. The only difference is the introduction of the $i \epsilon$-prescription which is necessary for convergence [20].

To summerize: In the operator quantization the operator $[\hat{\rho}, \hat{Q}]$ appears in order to make the inner products finite. It is not directly connected to the Hamiltonian operator. However, in the transition amplitudes it generally appears as an additional non-hermitian term to the Hamiltonian which due to the quantization rules that have to be imposed appears as an additional real term to the Hamiltonian in the path integral. This picture requires us to quantize Lagrange multipliers with indefinite metric states. However, sometimes they may be quantized with positive metric states if instead the variables which the gauge generators $\psi_{a}$ eliminate are quantized with indefinite metric states [9]. This is the case for propagators where the Hamiltonians may be interpreted as regularized real Hamiltonians.

An important issue in the path integral expressions (15) and (17) is the choice of boundary conditions. As is well known good physical properties are only obtained if certain Ward identities are satisfied and these identities follow from the imposed boundary conditions. The operator quantization yields apart from the above refine- 
ments of the path integrals also a connection between boundary conditions and the choice of gauge fixing. From the results of [7, 8] we find that the corresponding path integral to (28) requires the boundary conditions

$$
\pi_{a}=0, \quad \eta^{a}=0, \quad \bar{\eta}_{a}=0
$$

at the endpoints for $\rho=\mathcal{P}_{a} v^{a}$, and

$$
\psi_{a}=0, \quad \mathcal{P}_{a}=0, \quad \overline{\mathcal{P}}^{a}=0
$$

at the endpoints for $\rho=\bar{\eta}_{a} \chi^{a}$ for unimodular gauge groups. Possible choices of boundary conditions were discussed in [15] and the boundary conditions (32) are identical to the ones in (1.10) there and (33) is partly in agreement with (1.6-7) $\left(\pi_{a}=0\right.$ has to be replaced by $\left.\overline{\mathcal{P}}^{a}=0\right)$. Notice that both (32) and (33) are BRST invariant since the $|\phi\rangle$-states are BRST invariant in the general solutions (20).

\section{References}

[1] L. D. Faddeev and V. N. Popov, Phys. Lett. 25B, 29 (1967)

[2] C. Becchi, A. Rouet and R. Stora, Ann. Phys. (NY) 98, 287 (1976)

[3] E. S. Fradkin and G. A. Vilkovisky, Phys. Lett. 55B, 224 (1975)

[4] I. A. Batalin and G. A. Vilkovisky, Phys. Lett. 69B, 309 (1977)

[5] G. Curci and R. Ferrari, Nuovo Cimento 35A, 273 (1976)

[6] T. Kugo and I. Ojima, Phys. Lett. 73B, 459 (1978); Suppl. Prog. Theor. Phys. No 66 (1979)

[7] R. Marnelius, Nucl. Phys. B395, 647 (1993)

[8] R. Marnelius, Gauge fixing and abelianization in simple BRST quantization., ITP-Göteborg report 93-17 (1993) 
[9] R. Marnelius, Proper BRST quantization of relativistic particles., ITPGöteborg report 93-18 (1993)

[10] W. Pauli, Rev. Mod. Phys. 15, 175 (1943)

[11] D. G. Boulware and D. J. Gross, Nucl. Phys. B233, 1 (1984)

[12] M. Henneaux and C. Teitelboim, Quantization of Gauge Systems, Princeton University Press (Princeton, 1992)

[13] R. Marnelius, Nucl. Phys. B391, 621 (1993)

[14] R. Marnelius, Int. J. Mod. Phys. A5, 329 (1990)

[15] M. Henneaux, Phys. Rep. 126, 1 (1985)

[16] R. Marnelius, Nucl. Phys. B370, 165 (1992)

[17] C. Teitelboim, Phys. Rev. D28, 297 (1983)

[18] C. G. Torre, Class. Quant. Grav. 5, L63 (1988) and in New Perspectives in Canonical Gravity Lecture Notes by A. Ashtekar and invited contributors, Napoli, Italy, Bibliopolis, 1988.

[19] R. Marnelius and M. Ögren, Nucl. Phys. B351, 474 (1991)

[20] C. Teitelboim, Phys. Rev. D25, 3159 (1982); M. Henneaux and C. Teitelboim, Ann. Phys. 143, 127 (1982) 